\title{
Quality of Service in Wireless Networks*
}

\author{
Vittorio Bilò Adriano Di Pasquale Fabio Fioravanti Michele Flammini Luca Forlizzi \\ Francesco Lo Presti Giovanna Melideo Enrico Nardelli Alfredo Navarra Guido Proietti \\ Dipartimento di Informatica \\ Università di L'Aquila \\ Via Vetoio, 67010 L'Aquila, Italy
}

\begin{abstract}
In this paper we revise some of the most relevant aspects concerning the Quality of Service in wireless networks, providing, along the research issues we are currently pursuing, both the state-of-the-art and our recent achievements. More specifically, first of all we focus on network survivability, that is the ability of the network of maintaining functionality as a consequence of a component failure. Then, we turn our attention on data access and network services in a distributed environment. Finally, we analyze a basic network optimization task, that is routing design in wireless ATM networks.
\end{abstract}

\section{Introduction}

Wireless networks are becoming more and more ubiquitous in recent years, ranging from mobile analog and digital cellular telephony up to satellite broadcasting. Therefore, supporting a high Quality of Service (QoS) in delivering voice, video and data in this context has emerged as one of the most important challenge of the new century. In this paper we discuss some of the most relevant topics concerned with QoS in wireless networks, focusing our attention on aspects like survivability, data access and layout design.

The term network survivability has been used in the past to denote the demand of maintaining functionality as a consequence of a network component failure (like, for instance, the removal of a link or the disruption of a facility location) [30]. Indeed, it is important to prevent catastrophic situations where, as a consequence of a component failure, the QoS in the network is affected.

\footnotetext{
* This work has been partially supported by the CNR-Agenzia 2000 Program, under Grants No. CNRC00CAB8 and CNRG003EF8, and by the Research Project REAL-WINE, partially funded by the Italian Ministry of Education, University and Research.
}

Among the others, an approach which is emerging in the last period for addressing the above problem is based on a two-levels view of the network itself: (1) the "working network", i.e., the backbone where communication is carried out in the absence of failures, designed by optimizing any given objective function; (2) an "emergency network", that is, a set of inactive links, ready to be used to create alternative routes as soon as the working network undergoes some component failure affecting its functionality.

According to the above model, an emergency network should be as close as possible (both in terms of physical components and of communications protocol) to the primary network, disregarding, to some extent, the demand of maximal efficiency (with respect to the optimization function addressed by the primary network). A motivation behind this approach is that one prefers to pay in terms of efficiency, once that the switching to the emergency network is fast and painless. This is even more true whenever the failure which affected the network is transient, and then we expect to switch back to the primary one quite rapidly.

From a theoretical point of view, this gave rise to a set of pseudo-dynamic connectivity problems on graphs, i.e., the mathematical counterpart of network survivability. By virtue of the adopted strategy (i.e., the fast swapping from the primary to the emergency network through the activation of some selected links), the computational procedures aiming at implementing what sketched above are known with the name of swap algorithms. In the past, efficient swap algorithms have been developed in the context of the following classic (i.e., infrastructured) network topologies: minimum spanning trees [22], single source shortest paths trees [47], minimum diameter spanning trees [35, 46] and minimum biconnected spanning networks [31]. However, for wireless networks, very few has been done. In Section 2, we describe some preliminary achievements that move one step ahead in filling this gap.

For what concerns efficient data management in wireless networks, mobile computing technologies realize a ubiq- 
uitous information environment where "data-hungry" users equipped with low powered portable computers can retrieve local or remote information anywhere and at anytime. A portable computer can access data stored in a wired network via a base station by wireless communications. A base station and a portable computer communicate through a wireless channel. A fundamental method for users to access data through wireless channels is the so-called on-demand method, in which users send requests to get data. From one hand, we focused on the theoretical problem of defining a data model for the moving objects associated with the users [27]. On the other hand, we considered a promising computational framework for the distributed maintenance of data among the bases of the wireless networks, using Scalable Distributed Data Structures (SDDS) [39, 41, 42, 44]. These are access methods specifically designed to satisfy the high performance requirements of a distributed computing environment made up by a collection of computers connected through a high speed network. The main goal of an access method based on the SDDS paradigm is the management of very large amount of data, implementing efficiently -in terms of number of exchanged messages- standard operations (e.g., inserts, deletions, exact searches, range searches, etc.) and aiming at scalability, i.e., the capacity of the structure to keep the same level of performances while the number of managed objects changes [15, 16, 17, 18].

Another important issue related with distributed data is that of certification, i.e., the capability of an infrastructure for e-services to provide a non-repudiable proof of the actual provision of a service. This is a fundamental issue underlying the development of any infrastructure for e-commerce and mobile commerce [45]. Within this certification framework our group has focused on the trustability issue, i.e. the degree of confidence a user can have in the reliability of another user he/she want to enter in a commercial transaction with [11] and on the performance measurement issue [1].

In Section 3, we turn our attention to the above described data management problems, and we survey some recent results we obtained along this direction.

Concerning the last aspect we focus on, that is the design and management of the network backbone, a relevant issue to be considered is the determination of routing methods able to guarantee a good QoS. The ATM protocol for Broadband Integrated Service Digital Networks is used for the backbone wired connection among base stations of wireless networks, and in particular it is part of the current releases of UMTS. In order to achieve the stringent transfer rate requirements, it performs routing by means of dedicated hardware implementing very simple algorithms. As a consequence, the protocol is based on two types of predetermined routes in the network: virtual paths and virtual channels. Routing in virtual paths can be performed very efficiently by dedicated hardware, while a message passing from one virtual path to another one requires more complex and slower elaboration. The concatenation of the virtual paths that realize the connection between two given nodes of the networks forms the corresponding virtual channel. The virtual path layout problem [34, 6, 28] consists in choosing a suitable set of virtual paths, so as to guarantee a good QoS. Namely, in a virtual path layout there are restrictions on the following parameters:

i. the hop count, i.e., the number of virtual paths whose concatenation forms a virtual channel of the network; this parameter must be kept low and determines the efficiency of the routing strategy $[4,50,51]$; indeed, the routing is performed in a hardware fashion in all the nodes a message traverses, except when it reaches the end of a virtual path;

ii. the load, i.e., the number of virtual paths that share any physical link; indeed, the protocol imposes an upper bound on the space used at the nodes and this parameter determines the number of entries of the routing tables (see [10]).

In Section 4, we will discuss how this well-stated parameters interplay with other primary aspects emerging in wireless ATM, like mobility and survivability.

\section{Survivability of Wireless Networks}

As far as survivability aspects are concerned, the purpose of our research has been mainly the extension of the swap techniques to wireless networks. In this respect, the basic assumption is that component failures are transient, and therefore the swapping to the emergency network is temporary and must be fast and as less invasive as possible. Moreover, since sooner or later each component in the network may be subject to a malfunctioning, we adopt the approach of computing the "swapping" with respect to all the possible, non-overlapping failures. In this way, on one hand we amortize the computational effort, and on the other hand we detect in advance the most vital network components (i.e., those whose removal will affect the network functionality the most), so that preventive actions to strengthen them can be accomplished.

As a first step towards this direction, we focused our attention on radio communication networks, where mobility is not contemplated. More formally, a radio communication network is defined as a set $\mathcal{S}=\left\{s_{1}, \ldots, s_{n}\right\}$ of radio stations located on the $d$-dimensional space, $d \geq 1$, and which can transmit and receive messages via ether. If a station $s$ has a given transmission range $R(s)$, then and all the stations within this range can receive messages directly (or, in a single hop) from $s$. Hence, a range assignment for $\mathcal{S}$ is 
a real function $R: \mathcal{S} \rightarrow \mathbb{R}^{+}$assigning to each station $s_{i}$ a range $R\left(s_{i}\right)$. Since it is commonly assumed that the signal power falls as $1 /\left|s_{i}-s_{j}\right|^{\kappa}$, where $\kappa \geq 1$ is a constant parameter depending on the transmission environment, and given that in an ideal environment $\kappa=2$ (see for example [49]), it follows that the communication power needed to support a range $R\left(s_{i}\right)$ is $R\left(s_{i}\right)^{2}$. Hence, the cost of $R$ can be defined as

$$
\operatorname{Cost}(R)=\sum_{s_{i} \in S} R\left(s_{i}\right)^{2} .
$$

Given a range assignment $R$ for $\mathcal{S}$, the transmission graph induced by $R$ over $\mathcal{S}$ is defined as a directed graph $G_{R}=$ $(\mathcal{S}, A)$ where

$$
A=\bigcup_{s_{i} \in \mathcal{S}}\left\{\vec{e}=\left\{s_{i}, s_{j}\right\} \mid s_{j} \text { is within the range of } s_{i}\right\} .
$$

Then, the fundamental trade-off that has to be addressed by any resource allocation algorithm in wireless networks is the following: Find a range assignment of minimum cost such that the corresponding transmission graph satisfies a given property $\pi$.

The very basic property $\pi$ is connectivity, that is, we are asked to guarantee the capability for each station to reach any other remote station. In this case, intermediate stations (or gateways) need to be used, livening up a multi-hop transmission. In some contexts, such as in cellular networks, a multi-hop transmission relies on a set of fixed base stations: each base station has enough power to reach in a single hop all the other stations, and all the base stations are usually connected through a high-speed wired backbone infrastructure (e.g., optical fibers). Hence, for a station $s$ to communicate with a station $t$ in such a scenario, a message needs to reach, through a set of gateways (i.e., non-base stations), a base $b_{s}$ associated with $s$, which will then deliver the message to $t$. The maximum number of hops allowed, say $h$, can either be unbounded (i.e., $h=n-1$ ) or bounded, and this dramatically influences the total power assignment of the network. An optimal range assignment with bases and bounded $h$ is then a range assignment in the network such that each non-base station can reach a base in at most $h-1$ hops, and whose total power consumption is minimum.

Given an optimal range assignment, routing of messages in the network is established through an ad-hoc communication protocol, in which any station knows exactly both its closest gateway towards the associated base (we call this station its parent), and the set of stations whom it has to serve as a gateway (we call these stations its children). The number of children of a station is a vital design feature, since it determines the congestion, in terms of traffic load, of the station. Moreover, in radio networks, a high number of children creates interference phenomena. Hence, the station must be provided in advance with hardware and software facilities adequate to support its congestion.

As soon as a transient failure of either a non-base or a base station $s \in \mathcal{S}$ happens, this will result in a disconnection of the network itself (unless $s$ is not a gateway). In [33], we focused on failures of non-base stations, since base stations deserve a different treatment. Hence, whenever a nonbase station $s$ fails, all the stations in $\mathcal{S}$ that use $s$ to reach their associated base, will remain isolated. How to proceed then to guarantee the network functionality? According to the swap strategy, we should make the least amount of changes on the network. This has been done by altering the global state of the minimum number of stations. More precisely we provided a time efficient solutions to the following problem: Whenever a transient failure of a non-base station happens, swap to an emergency network in which the set of stations having their range changed or congestion increased is minimum, and, under the same number of changes, with the least total power consumption.

In [33], we restricted our attention to the case where the stations are spread on a line. This is the only case so far that the optimal range assignment can be computed efficiently [8]. Despite its apparent simplicity, this scenario results in an accurate model for several practical situations, for example, when the stations are located along a road or a railway. We showed that depending on the number and distribution of the children of the failed station, an efficient solution can always be found, based on updating 1 station in $O(h \log n)$ time, 2 stations in $O(n)$ time or 3 stations in $O(h n)$ time, all using $O(n)$ space, where $n$ is the number of stations.

Along the same direction as sketched above, we turned our attention to the 2-dimensional plane, and we faced the following two combinatorial optimization problems, which play a crucial role in wireless networking:

\section{Minimum-Energy Broadcast Routing} (MEBR): Given a source node $s \in \mathcal{S}$, find a minimum-cost range assignment $R^{*}$ such that the transmission graph $G_{R^{*}}$ contains a branching (directed spanning tree) rooted at $s$;

2. Minimum-Energy Complete Routing (MECR): Find a minimum-cost range assignment $R^{*}$ such that the transmission graph $G_{R^{*}}$ is strongly connected.

Unfortunately, it turns out that they are both NP-hard (see [9] and [7], respectively). However, they are approximable within a constant factor, through the computation of a minimum spanning tree (MST) of the complete weighted graph $G_{\mathcal{S}}^{(2)}=(\mathcal{S}, E)$, in which the edge costs are given by the square of the Euclidean distance between any pair of stations. More precisely, ranges are assigned as follows, respectively: 
1. For the MEBR-problem, direct the computed MST from the root towards the leaves, and assign to each node a range equal to the Euclidean distance of the farthest children in the directed MST;

2. For the MECR-problem, assign to each node a range equal to the Euclidean distance of the farthest adjacent node in the computed MST.

In this way, it turns out that the MEBR problem can be approximated within 12 [55], while the MECR problem can be approximated within 2 [37]. Additionally, the MST serves as an optimal solution [37] for the MINIMUMENERGY ALL-TO-ONE ROUTING-problem, defined as follows: Given a source node $s \in \mathcal{S}$, find a minimum-cost range assignment $R^{*}$ such that the transmission graph $G_{R^{*}}$ contains a branching oriented towards $s$. In this case, it suffices to direct the computed MST towards the source $s$, and to assign to each station a range equal to the Euclidean distance from the successor onto the path towards $s$.

Once established the usefulness of the MST structure for wireless networks, in [32] we have studied the following problem: Given a set of stations $\mathcal{S}$ in the 2-dimensional space, and given a range assignment $R: \mathcal{S} \rightarrow \mathbb{R}^{+}$induced by an MST of $G_{\mathcal{S}}^{(2)}$, find for every $s \in \mathcal{S}$ an MST of $G_{\mathcal{S}-s}^{(2)}$. We called this problem the All STATIONS REPLACEMENT (ASR) problem with input $\mathcal{S}$ and $R$, and we proved that it can be solved on a pointer machine in $\mathcal{O}(n)$ time and space, thus improving the previous known $\mathcal{O}(n \cdot \alpha(n, n))$ time bound holding for general sparse graphs [48], where $\alpha$ is the functional inverse of Ackermann's function. This has been obtained by exploiting the geometric properties of an MST of $\mathcal{S}$, which is actually a Euclidean MST. As far as we know, this is the first result which is expressly tailored for dealing with fault-tolerance in 2-dimensional radio networks, since previous papers were only focused on either designing of redundant communication protocols [38], or on the linear case [33].

In a quite similar scenario, but from a different point of view, we focused on the problem of monitoring the internal state of a communication network supporting mobile users, since guaranteeing QoS in this framework requires a prompt detection and reaction to system congestion and/or failures. Unfortunately, this problem is complicated by the fact that the mobile operator might use a third party network to interconnect the base stations, and the state of these might be not directly accessible by the mobile operator (bear in mind that, indeed, commercial factors often prevents organizations to share network performance data). To this end, we have investigated techniques to monitor the network topology and load by means of end-to-end traffic alone (e.g, traffic exchanged by the base stations) without access to the network operational information. In [13], we showed how end-to-end loss statistics can be used to reconstruct the network topology without prior information (an adaptive strategy which generalizes this approach has been presented in [12]). In [14], we also illustrated how to use the same endto-end traffic to characterize the entire network state, e.g., individual link loss and delay statistics. Currently, we are investigating how these techniques can be effectively deployed to support QoS. The main problem is to cope with potential scalability issues in the measurement, inference and result dissemination among participating nodes.

\section{Data Access and Wireless Network Services}

\subsection{Distributed data access}

Concerning algorithms for data location, we have considered the privileged access method for wireless networks, i.e., the on-demand one. In this setting, we concentrated on efficient SDDS access methods and on a variant of the SDDS model.

We first investigated structures able to efficiently manage range searches, nearest neighbor searches and ordering. This study is motivated as follows: The problem of finding efficient exact searches and insertions in SDDS is already solved by $\mathrm{LH}^{*}$ [41]. It provides a worst case cost of 4 messages for both operations. Despite this nice behavior, a range search can cause the visit of all the servers of the structure, even for a very small query range. $\mathrm{LH}^{*}$ has the same bad results for other operations involving the scan of multiple servers. Instead, search trees based SDDSs provide very good performance for range searches and related operations.

We first proposed an extension of the DRT [39] (Distributed Random Tree), an order preserving SDDS able to manage both mono-dimensional and multi-dimensional data, namely the DRT*. Then, in [16] we analyzed the amortized behavior of the DRT* and we proved that a sequence of $m$ requests of intermixed exact-searches and insertions over a DRT* starting with one empty server and ending with $n$ servers has a cost of $C(m, n)=$ $O\left(m \log _{(1+m / n)} n\right)$ messages. Such a result is obtained by adapting some of the techniques developed for the solution of the Set Union Problem [54]. Hence, in [20] we continued to analyze such a relation to find better performances. In fact, we showed that by adding to the DRT* strategy a correction algorithm just after each split of a server, we are able to improve the above cost to $O(m \cdot \alpha(m, n))$ messages, where $\alpha(m, n)$ is the classic inverse of the Ackermann function [53]. Due to the well known slow growth of the function $\alpha(m, n)$, we can assume to have $C(m, n) \simeq O(m)$ in realistic scenarios of SDDS made up by thousands or even millions of servers.

Still along this research line, we turned our attention to 
the distributed $B^{+}$-tree [3], the first order preserving SDDS with constant exact search worst-case cost, but at the price of a linear worst-case cost for insertion. In $[18,19]$, we further developed the technique presented in [3] with the major objective to keep logarithmic the worst-case cost of insertions. This allows to obtain the following results: (i) worst-case constant cost for exact searches and insertions that do not causes splits, namely 4 messages; (ii) worst-case logarithmic cost for insertions that causes splits; (iii) amortized almost constant cost for any single-key operations. Therefore, this was the first order preserving SDDS proposal achieving single-key performances comparable with the $\mathrm{LH}^{*}$, while continuing to provide the good worst-case complexity for range searches typical of order preserving access methods.

From the SDDS model point of view, we relaxed some hypothesis of the model, in order to provide a more realistic complexity measure of SDDS data structures. A widely used hypothesis in the SDDS model is to consider the cost of an operation as the number of messages exchanged by the sites. The real cost of a message, in terms of number of hops, is not accounted. This means that the graph associated with the underlying network is a complete graph. We relaxed this hypothesis and we provided a preliminary analysis the impact of the topology of the underlying network on the complexity of SDDSs. More precisely, in [21] we defined the problem of allocating servers in a network of workstations. Informally, given a set of already allocated servers $S$ in a network $G$, an overloaded server $s \in S$, in order to balance its load, may ask for the creation and placement of a new server $t$ in a node of $G$ not already occupied by another server. A given allocation of a set of servers $S$ in $G$ is evaluated in terms of the following two cost measures:

1. The maximum distance $d(s, t)$ in $G$ between pairs of servers $s, t$ such that $t$ is generated by $s$.

2. the diameter of $S$ in $G$, that is the maximum distance in $G$ between two servers of $S$.

The allocation problem is directly applicable to the SDDS context. Here, whenever a server $s$ is in overflow, that is it manages more data than its capacity, a split operation is performed: $s$ instantiates a new server $t$ and migrates to it approximatively half of its data. An algorithm solving the allocation problem (that we call allocation algorithm) with the above mentioned requirements is a basic component of the split operation procedure. The first requirement is important in order to minimize the startup cost of a new server, as large amount of data must be migrated spending a time proportional to the servers distance. The second one is important because the diameter of the graph is an upper bound on the cost of query and update messages in terms of time or length of the paths traversed in the network.

In realistic scenario, where the topology of the network is considered, the allocation problem cannot be trivially solved and allocation algorithms like the ones we developed become of fundamental importance for the determination of efficient SDDS operations. Therefore, given a bound on the diameter of the allocated servers, we concentrated on the determination of solutions that minimize the maximal distance between pairs of servers involved in a split operation. In particular, we provided optimal and nearly optimal approximation algorithms, both in the off-line setting, in which all the sequence of splits is known in advance, and in the on-line one, in which at each step a new server must be allocated without knowledge of the future splits.

\subsection{Network services}

A fundamental issue for a real uptake of electronic commerce and mobile commerce (m-commerce) regards trust among parties in authentication infrastructures: a reference entity $e$, before establishing an $\mathrm{m}$-commerce relation with a (previously unknown) target entity $t$, would like to know to which degree it can trust $t$.

We have investigated in [11] algorithmic techniques to efficiently establish trust degrees among entities. Trust relations can be modeled with a weighted directed graph, called trust graph, where the weight of a directed edge from $x$ to $y$ expresses the degree of $x$ 's trust in $y$. Then $e$ has to establish a trust path between itself and $t$ (i.e., an edge in the transitive closure of the trust graph) whose overall weight is the degree of its trust in $t$. There are two contrasting objectives: (i) the search should produce a path to $t$ providing a large enough degree of trust - otherwise the m-commerce relation cannot be established on a reliable enough base, (ii) the search process should be efficient, both in time spent and in computational resources used. Since searching the trust graph in an unrestricted manned can in general lead to a waste of time and computational resources, the search process has to be guided towards more promising directions of exploration. We have proposed in [11] efficient algorithms to find a trust path by building an incrementally larger user's viewpoint on the entire trust graph.

One of the hottest challenges in the digital government arena is the capability of providing good quality services to citizens. The critical issue is that for a given service a citizen usually interacts with a single provider, even if service supply and management requires coordination and cooperation among many autonomous organizations. This means that a single user request spreads in the underlying distributed information system and activates a number of information flows among organizations involved, with various roles and responsibility, in service provision. A main is- 
sue is how what is going on in the distributed system can be objectively monitored so that the service provider can $(i)$ understand and manage problems in the overall service supply process and (ii) certify quality of provided service. What makes this scenario especially complex is that, beyond technical aspects, any solution that wants to be successful has to comply with requirements of independence and autonomy of the various organizations involved. In [1] we tackled and solved this issue in real-world systems defined for the Italian Public Administration and we argue that our solution can provide a reference architecture to deal with this kind of problems.

\section{Layout Design in Wireless ATM Networks}

The integration of wireless and ATM (WATM) networks is emerging as one of the most promising approaches able to support users mobility while maintaining the quality of service offered by the classical ATM. This combination occurs at different levels and yields different scenarios, such as End-to-End WATM and WATM Interworking, applied respectively to create new wireless networks with ATM virtual channels extending until the mobile terminals and at a more external level for interconnecting different existing wireless subnets [29]. In both scenarios, the mobility facility requires the efficient solution of several problems, such as handover (users movement), routing, location management, connection control and so forth. A detailed discussion of these and other related issues can be found in [2, 29, 5, 52]. In [24] we have proposed an extension of the basic ATM model of $[34,6]$ able to combine quality of service and mobility aspects in wireless ATM. In this model a subset of the nodes of the network represents base stations and users are allowed to move between themaccording to an adjacency graph expressing adjacencies of the stations in the geographic space Such a graph in general can differ from the physical topology of the infrastructured network. For instance, in nowadays cellular systems like GSM, the physical graph $G$ is a tree, stations correspond to its leaves and the adjacency graph is an hexagonal grid (see for instance [43]). Standard ATM layouts must be constructed in order to establish a different VC for each station, but besides the hop count and the load, their performance is evaluated by means of a further parameter, the virtual channel distance, that measures the time needed to reconstruct virtual channels during hand-off phases, that is when mobile terminals switch between adjacent stations. More precisely, the distance between the virtual channels of two adjacent nodes is equal to the number of VPs that must be deleted and added to one VC in order to obtain the other one. In order to make the rerouting phase imperceptible to users and thus to obtain a sufficient quality of service, the maximum distance between two virtual channels must be maintained as low as possible. Therefore, a natural combinatorial problem arises in which suitable trade-offs must be determined between the different performance measures.

In [24] it has been shown that the layout construction problem is intractable, that is NP-hard. Moreover, optimal layout constructions are given when the physical and adjacency graphs are coincident and correspond to basic interconnection networks, such as chains and rings. Such results hold under the assumption that all the VCs induce shortest paths in the underlying network. In [25] we consider the determination of WATM layouts for chains in the nonshortest path case in which the lengths of the paths induced by the VPs is not constrained. We first show that deciding the existence of a layout with maximum hop count $h$, load $l=1$ and distance $d=1$ is NP-complete even when the adjacency graph is a chain of base stations with the source coinciding with one of its endpoints. Moreover, such a hardness result is extended to every fixed value of $d$. We then consider the case in which the physical and adjacency graph coincide with chains and provide optimal layout constructions for $d \leq 2$. Finally, optimal layout constructions are obtained also for any $d$ within the class of the so-called canonic layouts, that so far have been always shown to be the optimal ones.

\section{Conclusions}

In this paper, we have revised some of the most relevant aspects concerning the QoS in wireless networks, that is survivability, data access and layout design, by providing both the state-of-the-art and the research issues we are currently pursuing.

In the future, concerning the survivability topic, we plan to extend our results to other typical network topologies (e.g., linear, linear with bases, grids, 2-dimensional, etc.), under various general assumptions (e.g., number of hops bounded or unbounded, with or without interferences, etc.), and with respect to different communication paradigms (e.g., one-to-one, all-to-one, broadcast, multi-broadcast, etc.), always by letting unaltered the basic criteria of minimizing the number of range changes. Moreover, we plan to estimate the quality of the swap solutions as compared to the optimal ones, as computed from scratch in the residual network.

Concerning data management, we will concentrate on indexing techniques for the broadcast data access method, in which the users of the wireless network retrieve their data by just listening to a certain channel on which a server broadcasts information. In the past, some indexing techniques have been proposed to speed-up access time and reduce power consumption under this scenario [36, 40]. However, these schemes were only concerned with some simple 
forms of hashing and on uniform data access probabilities. Moreover, the majority of the approaches have only considered the case of a single broadcast channel. Hence, we foresee the exploitation of multiple channels in order to achieve better access and tune-in times for the mobile units.

Finally, concerning future research directions for WATM networks, one important question left open in [25] is if the family of the canonic layouts contains optimal layouts for $d>2$. Even if not claimed explicitly, our constructions show that this is true for $d \leq 2$ and the previous results shown in the literature seem to confirm this conjecture. Moreover, it would be nice to extend our results to more general topologies and to the case in which the physical and adjacency graphs are not coincident. Another worth investigating issue is the extension to other communication patterns like all-to-all, that is when connections can be established between every pair of mobile users. It would be nice to investigate the approximability of the layout construction problem under the shortest paths assumption and when $d>1$ in case of non-shortest paths. Finally, besides the wireless context, our model can be applied also to address survivability issues in ATM. In this case, virtual channels must be reconstructed as a consequence of link failures, and again an important issue is the determination of layouts maintaining a small distance between basic and replacement channels.

\section{References}

[1] F. Arcieri, F. Fioravanti, R. Giaccio, E. Nardelli and M.Talamo, Certifying performance of cooperative services in a digital government framework, in Proc. of the 2003 International Symposium on Applications and the Internet, 2003.

[2] B.A. Akyol and D.C. Cox, Rerouting for handoff in a wireless ATM network, Proc. of the IEEE International Conference on Universal Personal Communications, 1996.

[3] Y. Breitbart and R. Vingralek, Addressing and balancing issues in distributed $\mathrm{B}^{+}$-trees, 1st Workshop on Distributed Data and Structures (WDAS'98), 1998, Carleton Scientific Press.

[4] J. Burgin and D. Dorman, Broadband ISDN resource management: the role of virtual paths, IEEE Communications Magazine 29, 1991, 44-48.

[5] C. Chrysostomou, A. Pitsillides and F. Pavlidou, A survey of wireless ATM handover issues, Proc. of the International Symposium of $3 G$ Infrastructure and Services, 3GIS, 2-3, 2001, 34-39.

[6] I. Cidon, O. Gerstel and S. Zaks, A scalable approach to routing in ATM networks, Proc. WDAG'94, Vol. 857 of Lecture Notes in Computer Science, Springer, 209-222.

[7] A.E.F. Clementi, P. Penna and R. Silvestri, Hardness results for the power range assignment problem in packet radio net- works, Proc. APPROX'99, Vol. 1671 of Lecture Notes in Computer Science, Springer, 197-208.

[8] A.E.F. Clementi, A. Ferreira, P. Penna, S. Perennes and R. Silvestri, The minimum range assignment problem on linear radio networks, Proc. ESA 2000, Vol. 1879 of Lecture Notes in Computer Science, Springer, 143-154.

[9] A.E.F. Clementi, P. Crescenzi, P. Penna, G. Rossi and P. Vocca, On the complexity of computing minimum energy consumption broadcast subgraphs, Proc. STACS'01, Vol. 2010 of Lecture Notes in Computer Science, Springer, 121131.

[10] R. Cohen and A. Segall, Connection management and rerouting in ATM networks, Proc. IEEE INFOCOM'94, 184-191.

[11] E. Di Vito, P. Inverardi and G.Melideo, A context-aware approach to infer trust in public key infrastructures, in Proc. of the International Workshop on Certification and Security in E-Services (CSES'02), 103-116.

[12] N.G. Duffield, J. Horowitz and F. Lo Presti, Adaptive Multicast Topology Inference, Proc. IEEE Infocom 2001, 16361645.

[13] N.G. Duffield, J. Horowitz, F. Lo Presti and D.Towsley, Multicast Topology Inference from End-to-end Loss, IEEE Transactions on Information Theory, 48(1), 2002, 26-45.

[14] N.G. Duffield, F. Lo Presti, V. Paxson and D. Towsley, Inferring Link Loss Using Striped Unicast Probes, Proc. IEEE Infocom 2001, 915-923.

[15] A. Di Pasquale and E. Nardelli, Distributed searching of $k$ dimensional data with almost constant costs, Proc. ADBISDASFAA 2000, Vol. 1884 of Lecture Notes in Computer Science, Springer, 239-250.

[16] A. Di Pasquale and E. Nardelli, An amortized lower bound for distributed searching of $k$-dimensional data, Proc. WDAS 2000, Carleton Scientific Press, in press.

[17] A. Di Pasquale and E. Nardelli, Scalable distributed data structures: a survey, Proc. WDAS 2000, Carleton Scientific Press, in press.

[18] A. Di Pasquale and E. Nardelli, A very efficient order preserving scalable distributed data structure, Proc. Int. Conf. on Database and Expert Systems Applications (DEXA 2001), Vol. 2113 of Lecture Notes in Computer Science, Springer, 186-199.

[19] A. Di Pasquale and E. Nardelli, ADST: An order preserving scalable distributed data structure with constant access costs, in Proc. 28th Annual Conference on Current Trends in Theory and Practice of Informatics (SOFSEM 2001), Vol. 2234 of Lecture Notes in Computer Science, Springer, 211-222.

[20] A. Di Pasquale, E. Nardelli and G. Proietti, An improved upper bound for scalable distributed search trees, in Proc. Workshop on Distributed Data and Structures (WDAS 2002).

[21] A. Di Pasquale, M. Flammini and E. Nardelli, Allocation problems in scalable distributed data structures, in Proc. Workshop on Distributed Data and Structures (WDAS 2002). 
[22] B. Dixon, M. Rauch and R.E. Tarjan, Verification and sensitivity analysis of minimum spanning trees in linear time, SIAM Journal on Computing 21(6), 1992, 1184-1192.

[23] T. Eilam, M. Flammini and S. Zaks, A complete characterization of the path layout construction problem for ATM networks with given hop count and load, Parallel Processing Letters 8(2), 1998, 207-220.

[24] M. Flammini, G. Gambosi and A. Navarra, Dynamic layouts for wireless ATM, Technical Report 4616, MASCOTTE Project, I3s-CNRS/INRIA/Université de Nice-Sophia Antipolis, France, 2002.

[25] M. Flammini, G. Gambosi and A. Navarra, Wireless ATM Layouts for Chain Networks, Proceedings of this workshop, 2003.

[26] M. Flammini, E. Nardelli and G. Proietti, ATM layouts with bounded hop count and congestion, Distributed Computing 14(2), 2001, 65-73.

[27] L. Forlizzi, R.H. Güting, E. Nardelli and M. Schneider, A data model and data structures for moving objects databases Proc. SIGMOD 2000, 319-330.

[28] L. Gasieniec, E. Kranakis, D. Krizanc and A. Pelc, Minimizing congestion of layouts for ATM networks with faulty links, Proc. MFCS'96, Vol. 1113 of Lecture Notes in Computer Science, Springer, 1996, 372-381.

[29] J.D. Gibson, The Mobile Communications Handbook, Second Edition, CRC Press in cooperation with IEEE Press, 1999.

[30] M. Grötschel, C.L. Monma and M. Stoer, Design of survivable networks, in: Handbooks in OR and $M S$, Vol. 7, Elsevier, 1995, 617-672.

[31] A. Galluccio and G. Proietti, Polynomial time algorithms for edge-connectivity augmentation problems, Algorithmica, to appear.

[32] C. Gaibisso, G. Proietti and R.B. Tan, Optimal MST maintenance for transient deletion of every node in planar graphs, 2002, submitted for publication.

[33] C. Gaibisso, G. Proietti and R. Tan, Efficient management of transient station failures in linear radio communication networks with bases, Proc. ARACNE'01, Vol. 12 of Proceedings in Informatics, Carleton Scientific, 37-54.

[34] O. Gerstel and S. Zaks, The virtual path layout problem in fast networks, Proc. PODC'94, 235-243.

[35] G.F. Italiano and R. Ramaswami, Maintaining spanning trees of small diameter, Algorithmica 22(3), 1998, 275-304.

[36] T. Imielinski, S. Viswanathan and B.R. Badrinath, Power efficient filtering of data on air, Proc. EDBT'94, 245-258.

[37] L.M. Kirousis, E. Kranakis, D. Krizanc and A. Pelc, Power consumption in packet radio networks, Proc. STACS'97, Vol. 1200 of Lecture Notes in Computer Science, Springer, 363374.

[38] E. Kranakis, D. Krizanc and A. Pelc, Fault-tolerant broadcasting in radio networks, Proc. ESA'98, Vol. 1461 of Lecture Notes in Computer Science, Springer, 283-294.
[39] B. Kroll and P. Widmayer, Distributing a search tree among a growing number of processor, Proc. SIGMOD'94, 265-276.

[40] W.C. Lee and D.L. Lee, Using signature techniques for information filtering in wireless and mobile environments, Journal on Distributed and Parallel Databases 4(3), 1996, 205 227.

[41] W. Litwin, M.A. Neimat and D.A. Schneider, $\mathrm{LH}^{*}$-Linear hashing for distributed files, Proc. SIGMOD'93, 327-336.

[42] W. Litwin, M.A. Neimat and D.A. Schneider, RP* - A family of order-preserving scalable distributed data structure, Proc. $V L D B^{\prime} 94,342-353$.

[43] M. Mouly and M.B. Pautet, The GSM System for Mobile Communications. Cell\& Sys, 1993.

[44] E. Nardelli, F. Barillari and M. Pepe, Distributed searching of multi-dimensional data: a performance evaluation study, Journal of Parallel and Distributed Computation 49, 1998, 111-134.

[45] E. Nardelli, S. Posadziejewski and M. Talamo, Certification and security in E-Services: from E-Government to EBusiness, Proc. IFIP 2002 17th World Computer Congress, Aug.02, Montreal, Canada, Kluwer Academic Publisher.

[46] E. Nardelli, G. Proietti and P. Widmayer, Finding all the best swaps of a minimum diameter spanning tree under transient edge failures, Journal of Graphs Algorithms and Applications 5(5), 2001, 39-57.

[47] E. Nardelli, G. Proietti and P. Widmayer, How to swap a failing edge of a single source shortest paths tree, Proc. COCOON'99, Vol. 1627 of Lecture Notes in Computer Science, Springer, 144-153.

[48] E. Nardelli, G. Proietti and P. Widmayer, Maintaining a minimum spanning tree under transient node failures, Proc. ESA 2000, Vol. 1879 of Lecture Notes in Computer Science, Springer, 346-355.

[49] K. Pahvalan and A. Levesque, Wireless information networks, Wiley-Interscience, New York, 1995.

[50] K.I. Sato, S. Ohta and I. Tokizawa, Broadband ATM network architecture based on virtual paths, IEEE Transactions on Communications 38(8), 1990, 1212-1222.

[51] Y. Sato and K.I. Sato, Virtual path and link capacity design for ATM networks, IEEE Journal of Selected Areas in Communications 9(1), 1991, 104-111.

[52] D. Sobirk and J.M. Karlsson, A survey of wireless ATM MAC protocols, Proc. of the International Conference on the Performance and Management of Complex Communication Networks (PMCCN '97). Chapman \& Hall, 1997.

[53] R.E. Tarjan, Efficiency of a good but nonlinear set union algorithm, J. Assoc. Comput. Mach., 22(2), 1975, 215-225.

[54] J. Van Leeuwen and R.E. Tarjan, Worst-case analysis of set union algorithms, J. Assoc. Comput. Mach., 31(2), 1984, 245-281.

[55] P.J. Wan, G. Calinescu, X.Y. Li and O. Frieder, Minimumenergy broadcast routing in static ad hoc wireless networks, Proc. INFOCOM'01, 1162-1171. 